\title{
Study of a circular Gaussian transition in an optical speckle field
}

\section{Isabelle Bergoënd}

\section{Xavier Orlik \\ xavier.orlik@onera.fr}

Eric Lacot

\begin{abstract}
Onera Centre de Toulouse, Département d'Optique Théorique et Appliquée, 2 avenue Edouard Belin, BP 74025, 31055 Toulouse cedex 4, France

Onera Centre de Toulouse, Département d'Optique Théorique et Appliquée, 2 avenue Edouard Belin, BP 74025, 31055 Toulouse cedex 4, France

Laboratoire de Spectrométrie Physique (UMR 5588), Université Joseph Fourier de Grenoble, BP87, 38402 St-Martin d'Heres Cedex, France
\end{abstract}

We propose a numerical approach to study optical speckle fields generated by various conditions of surface roughness and illumination, without any a priori assumption concerning the properties of the scattered field. By overcoming the limitations imposed by the use of the central limit theorem, we perform the study of the whole circular Gaussian transition undergone by the speckle field when varying the surface roughness and the size of the laser illumination. We focus our attention on the speckle contrast, the degree of circularity and the degree of asymmetry, including their radial dependence. [DOI: 10.2971/jeos.2008.08028]

Keywords: circular Gaussian transition, random rough Gaussian surfaces, speckle, circularity, asymmetry

\section{INTRODUCTION}

Laser speckle [1] may be considered as the ultimate limiting noise in optical imaging measurements with coherent light. However, under certain conditions, it also carries information about the characteristics of the illuminated scattering object. Since the beginning of experimentations with lasers, a lot of work has been achieved to understand speckle and to predict its statistical properties, either for noise reduction, or for surface roughness measurement. Goodman [2] has studied the first and second-order statistics of fully and partially developed speckle. This study has been performed under the hypothesis of a diameter of illumination broad enough to include a sufficient number of scattering contributions so that the central limit theorem could be applied. Then, many investigations have been carried out to obtain the speckle statistics in cases of fewer illuminated scattering cells giving rise to non Gaussian speckle, but always with restricting hypothesis. Especially, Pedersen [3] has studied the evolution of the speckle contrast with a decreasing number of scattering cells inside the illumination beam. The surface was described as a set of independent and horizontal random facets and the results were limited to the specular direction. Moreover, Jakeman and Pusey [4] have calculated theoretically the moments of intensity of non-Gaussian speckle but only in the hypothesis of a deep random phase screen.

When the surface roughness becomes lower than the wavelength, the breaking of the property of circularity concerning the distribution of the scattered field in the complex plane gives rise to a different statistical regime that can be very useful to characterize the surface. Such a scattered field has been modelized by Ohtsubo and Asakura [5] using the sum of a specular component and a diffuse component of zero mean.
They have especially inferred that, in the conical specular region around the optical axis, the circularity of the field was lost. Moreover, a theoretical work from Uozumi and Asakura [6] has presented the probability density functions of noncircular speckle fields but with the assumption of a large number of scatterers illuminated. Theoretical works dealing with non-Gaussian speckle and roughness lower than the wavelength can be found but are generally limited to a roughness lower than one third of the wavelength $[7,8]$.

Finally, to our knowledge, there are only few theoretical works dealing with non-Gaussian speckle for roughness values included in the range $[\lambda / 3, \lambda]$, in spite of the potential of this domain to characterize surfaces properties, as will be shown in this work. Among them, we can find the work of Fujii et al. [9] that have first used computer simulations to deduce speckle properties. They have calculated the intensity distribution and the contrast variation of the speckle field for various symmetrical surface profiles taking into account the number of correlation cells included in the point spread function of their optics. Later, the same team has completed this work [10] by enlarging the previous study to both asymmetrical Gaussian random surfaces and Gaussian random surfaces combined with a sinusoidal variation. However, these two previous studies were limited to surfaces modelized as one-dimensional objects. Uozumi and Azakura, in a further work [11], have then derived the probability density function of intensity for speckles produced by weak diffusers with very few illuminated correlation cells in the case of a 2D surface. However, this surface was described by a discrete cell model that assumed statistical independence between each dephasing cell and did not take into account any correlation of the 
surface. At the same time, Fujii [12], assuming a Gaussian distribution of the surface heights distribution, has performed an analytical study of the contrast for non-Gaussian speckle scattered from a low surface roughness and more recently, Hansen et al. [13] have used the ABCD matrices formalism to calculate analytical functions of first-order statistics concerning such partially developed speckle. However, these two latter works needed an approximation concerning the correlation function of the optical scattered field and have limited their results on the optical axis.

Finally, the statistical characteristics of speckle fields in the circular and Gaussian transition, and the effect of their coupling are still poorly described at this time. On a practical point of view, such new information is expected to participate to the improvement of absolute surface roughness and correlation length measurements by speckle analysis [14]-[16]. Especially, instead of characterizing the surface using a constant illumination area with various optical apertures as performed in the latter reference for random surface samples with Gaussian correlation, we provide here qualitative and quantitative results about some speckle statistics that give new insights concerning a surface characterization method based on a varying illumination area of the sample and that doesn't require any detection optics. For this purpose, we propose here a numerical approach to complete the missing data concerning partially developed non-Gaussian speckle fields generated by random rough surfaces. The model proposed here, subjected to the only paraxial approximation, is able to provide speckle statistics for a large range of surface roughness and illumination conditions, including the ones where the central limit theorem can't be applied. After a brief revue concerning the properties of a circular Gaussian speckle field in Section 2, a description of our approach is proposed in Section 3. In Section 4, we first perform some calculation about the on axis speckle contrast in a particular Gaussian transition in far field in order to compare our results with previous theoretical and experimental works. Finally, we propose in Section 5 the study of the circular Gaussian transition of a speckle field through the contrast, the degree of circularity and the degree of asymmetry, including their radial dependence. We have chosen to perform this study in near field in order to exhibit the properties of asymmetry of the complex field.

\section{BRIEF REVIEW OF SOME SPECKLE PROPERTIES}

Let us consider the propagation in free space of a monochromatic wave completely polarized that is dephased by the scattering on a rough material modeled by a deep phase screen. At an observation point, the complex amplitude of the scattered field $\tilde{E}(x, y, z)$ can be interpreted as a sum of many independent contributions. In the hypothesis that each contribution has an amplitude and a phase independent of each other and independent of the other contributions, and that the surface roughness is considered to induce an uniformly distributed dephasing of the incident field over $[0,2 \pi]$, Goodman [2] has shown that, in the case of a sufficient number of contributions, $\operatorname{Re}\{\tilde{E}\}$ and $\operatorname{Im}\{\tilde{E}\}$ are two random variables that have zero means, equal variances and that are uncorrelated. Further- more, according to the central limit theorem, if the number of contributions tends towards infinity, the real and imaginary part of the field both approach a Gaussian distribution. Their joint probability density function then follows asymptotically the equation :

$$
P(\operatorname{Re}\{\tilde{E}\}, \operatorname{Im}\{\tilde{E}\})=\frac{1}{2 \pi \sigma^{2}} \exp \left[-\frac{\operatorname{Re}\{\tilde{E}\}^{2}+\operatorname{Im}\{\tilde{E}\}^{2}}{2 \sigma^{2}}\right]
$$

where $\sigma$ is the standard deviation of this Gaussian distribution. Then, the field follows the statistics of a complex circular Gaussian random variable and the contrast of such completely developed speckle is :

$$
C=\frac{\sigma_{I}}{\langle I\rangle}=1
$$

where $\sigma_{I}$ is the standard deviation of the intensity and $\langle I\rangle$ the mean intensity.

In addition to the absence of correlation between the real and imaginary part of the complex field, the property of circularity refers to the conditions of zero means and equal variances of its real and imaginary parts, which indicate that the lines of equiprobability density of the field are circles around zero in the complex plane. This description of the speckle is valid for a very rough surface (standard deviation of heights distribution superior to the illuminating wavelength $\lambda$ ) in the case of many scatterers illuminated. However, dephasings of the field due to topography of rough surfaces are not properly described by random uncorrelated heights distribution and it is necessary to introduce a correlation between them characterized by a correlation length and a corresponding correlation cell in two dimensions. When only a small number of these cells are illuminated, the central limit theorem can no longer be applied. Consequently, the probability density function of the complex field does not follow Eq. (1) anymore. Then, the contrast can reach values higher than 1 because of the sporadic presence of very bright spots.

Surfaces with a roughness lower than the wavelength usually give rise to non-circular speckle because the phases of the scattered contributions are not uniformly distributed over $[0,2 \pi]$. Then, the mean value of the complex scattered amplitude can depart from zero and the corresponding lines of equiprobability of the field in the complex plane can describe ellipses or more complicated structures as will be shown in this work. Such situations give rise to a remaining specular component in the speckle field, decreasing the observed contrast.

\section{PRESENTATION OF THE NUMERICAL MODEL}

The model used in this paper consists first in a random rough surface generation and then in the calculation of the corresponding scattered field at a given distance in the case of a Gaussian beam illumination. For a constant set of roughness parameters and illumination conditions, by iterating this sequence a sufficient number of times (from 5,000 to 12,000 times depending of the complex field distribution in the observation plane), all the statistical parameters of the generated speckle field that we have studied reach a stable value with a weak 
remaining oscillation that will be represented as uncertainty bars on the following graphs. We have chosen a correlated Gaussian surface that exhibits a Gaussian height distribution. This surface profile, intensively studied in the past decades, allows the validation of our approach by comparison with previous experimental and theoretical studies at particular regions of the circular Gaussian transition where data are available. Moreover, it represents a sufficiently simple model for a good understanding of the coupling between the circular and Gaussian transitions.

The discretized and correlated profile $\mathcal{S}(\xi, \eta)$ of the random surfaces that will be used to generate the random phase screens are calculated by the following equation [17] :

$$
\mathcal{S}(\xi, \eta)=F T^{-1}[\sqrt{F T[\mathcal{A}(\xi, \eta)]} \cdot F T[X(\xi, \eta)]]
$$

where :

$$
\mathcal{A}(\xi, \eta)=H_{R M S}^{2} \exp \left[-\frac{\xi^{2}+\eta^{2}}{L_{c}^{2}}\right]
$$

with :

- $H_{R M S}$ the standard deviation of the surface heights distribution that will be simply referred as "roughness" in the forthcoming sections

- $L_{c}$ the correlation length of the surface heights distribution

- $X(\xi, \eta)$ an uncorrelated Gaussian random function with zero mean and standard deviation of unity

FT denotes here a discrete bidimensional Fourier Transform.

The initial scalar field characterized by a Gaussian amplitude distribution and a wavelength $\lambda=632 \mathrm{~nm}$ impacts the surface parallel to its normal. Its waist $\omega_{0}$ is chosen to be located on the mean level of the surface. The field undergoes the dephasing associated with the random correlated Gaussian surface and the scattered field at the distance $z$ in the transverse plane $(x, y)$ is then calculated using the Fresnel diffraction integral [18]:

$$
\tilde{E}_{d}(x, y, z)=\iint_{\mathcal{S}} \tilde{E}(\xi, \eta) \cdot \tilde{\mathcal{G}}(x-\xi, y-\eta, z) d \xi d \eta
$$

with $\tilde{\mathcal{G}}$ the propagator in the paraxial approximation :

$$
\tilde{\mathcal{G}}(x-\xi, y-\eta, z)=\frac{e^{i k z}}{i \lambda z} \cdot \exp \left[\frac{i k}{2 z}\left((x-\xi)^{2}+(y-\eta)^{2}\right)\right]
$$

and $\tilde{E}(\xi, \eta)$ the initial Gaussian beam of maximum amplitude unity that has been dephased according to the correlated surface profile $\mathcal{S}(\xi, \eta)$ :

$$
\tilde{E}(\xi, \eta)=\exp \left[-\frac{\xi^{2}+\eta^{2}}{\omega_{o}^{2}}\right] \cdot \exp [i k \mathcal{S}(\xi, \eta)]
$$

The previous calculation is performed using Fourier Transforms :

$$
\tilde{E}_{d}(x, y, z)=F T^{-1}[F T[\tilde{E}(\xi, \eta)] . F T[\tilde{\mathcal{G}}(x-\xi, y-\eta, z)]]
$$

As our aim is to study the circular Gaussian transition of a speckle field, we do not take into account more complicated phenomena such as volume or multiple surface scattering.

For the analysis of the scattered field, we define at a distance $z$ in an observation plane parallel to the surface, square Regions Of Interest (ROIs) that are smaller at least by a factor of 10 compared to the speckle grain size and that are used to characterize the complex field at different places. Concerning the study in Section 5 the first ROI is located on the optical axis at the distance $0.75 z_{R}$ from the surface, $z_{R}$ being the Rayleigh distance. Other ROIs are used to perform a radial study at the same longitudinal distance : the step between two consecutive radial ROIs has been calculated every $0.1 \omega$, where $\omega$ is the size that the beam would have at the longitudinal distance of observation without any rough surface. In consequence, each realization of a rough surface with the calculation of the corresponding scattered field gives a set of radial values for the real and imaginary parts of the field. We would like to emphasize that due to our choice about the size of the ROI compared to the grain size, the statistical parameters of the scattered speckle field are not defined for each realization of surface but are deduced from the whole realizations, each of them bringing one sample value of the complex scattered field for a given radial distance. Let $I_{n}(r)$ be the intensity measured at the radial distance $r$ in the $n^{\text {th }}$ realization and $N$ the number of realizations. The mean intensity and the standard deviation of the intensity are then calculated by :

$$
\langle I(r)\rangle=\frac{1}{N} \sum_{n=1}^{N} I_{n}(r)
$$

and

$$
\sigma_{I}(r)=\sqrt{\frac{1}{N-1} \sum_{n=1}^{N}\left(I_{n}(r)-\langle I(r)\rangle\right)^{2}}
$$

Then we can calculate the contrast as $C=\sigma_{I} /\langle I\rangle$. This method used for the determination of the statistical parameters of the speckle field is different for example from the method of Fujii et al. [9], who made ensemble averages of the contrast measured inside each image. In our case, it is representative of an experimental setup where a photodiode (with a detection area much smaller than the grain size) is used to measure the intensity while the surface is scanned. Moreover, the advantage of this method is that the measured data are independent of the grain size, which is not always the case when the statistical parameters are deduced from an ensemble of measurements inside the same image.

\section{COMPARISON WITH PREVIOUS RESULTS IN FAR FIELD: ON-AXIS SPECKLE CONTRAST IN A GAUSSIAN TRANSITION FOR A LOW ROUGHNESS SURFACE}

Before studying the circular Gaussian transition in near field, we propose here some calculations in far field concerning a Gaussian transition that has been previously studied from a ground glass sample. In this experiment performed by P. J. Chandley et al. [19], the Gaussian transition has been described by enlarging the beam waist size from $5 \mu \mathrm{m}$ to $100 \mu \mathrm{m}$ 
for a constant correlation length of the surface height fluctuations of $20 \mu \mathrm{m}$. In Figure 1, we compare their experimental results concerning the on-axis speckle contrast for a roughness value of $\lambda / 3$ at $\lambda=632 \mathrm{~nm}$ with both our results and the theoretical ones from H. M. Escamilla [7]. This latter model uses a multinomial expansion of the second moment of intensity in powers of the random phase fluctuation and is available for low roughness values and for on-axis positions only due to an accumulation of errors during the computation that limits the order of this expansion. However, this model is expected to be efficient for the description of the experiment cited above. We observe that the three on-axis contrast curves exhibit a similar behaviour with a common value of the maximum for a laser spot radius of about $1.5 L_{c}$. Moreover, we notice a good matching between the theoretical data from $\mathrm{H}$. M. Escamilla and our numerical results until the illumination beam waist reaches $80 \mu \mathrm{m}$ where our contrast becomes slightly higher, and exhibits a slope very similar to experimental data. As mentioned by H. M. Escamilla, the experiment that uses liquids of various refraction indices sandwiched between the ground glass and a polished slide to allow measurements with different standard deviation of the wavefront height, can undergo multiple reflections that can have a significant influence on the measured contrast. On the other hand, we see that the stronger difference between theoretical curves and experiments occur in the highly non Gaussian regime, where only $1 / 4$ of a correlation length is illuminated. This could be explained by the fact that a such very localized illumination of the surface exhibits a more detailed microscopic structure of the ground glass that is no more efficiently described by a Gaussian correlated surface. In favour of this hypothesis, it has been shown, with atomic force microscopy $[20,21]$ that ground glass generally exhibits a surface morphology including fractal characteristics.

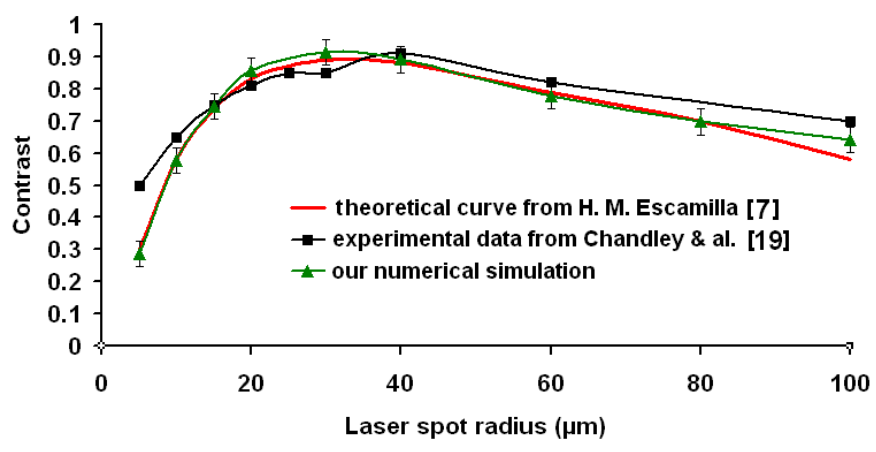

FIG. 1 Speckle contrast versus laser spot radius : comparison between our results, an analytical calculation [7] and experimental data [19]. $L_{c}=20.3 \mu \mathrm{m}, H_{R M S}=\frac{\lambda}{3}$, $\lambda=632 \mathrm{~nm}$.

\section{THE CIRCULAR GAUSSIAN TRANSITION IN NEAR FIELD}

As pointed out in the introduction, a part of the circular Gaussian transition is still poorly described at this time. On the experimental point of view, it is difficult to have samples with the same correlation length of the surface heights distribution but with increasing surface roughness values. Moreover, the use of liquids with different refraction indices for changing the roughness value of the surface compared to the wavelength is suspected to induce multiple reflections. On the theoretical point of view, the fact that the central limit theorem is no more usable when only a few correlation cells are illuminated has strongly limited the theoretical studies in this range and most of them are submitted to approximations. In consequence, we propose in this section to give results concerning the speckle contrast, the degree of circularity and the degree of asymmetry in the circular Gaussian transition, in the only paraxial approximation. Radial dependence of the statistical parameters will be also given. The longitudinal distance of observation has been chosen in the near field of the illuminating Gaussian beam because this region is of particular interest due to the fact that the speckle field exhibits a strong correlation between its real and imaginary part [6].

We have calculated with the model described in Section 3 the complex field at the distance $z=0.75 z_{R}$ from the surface with standard deviations of the surface heights distribution $H_{R M S}$ of $\lambda / 10, \lambda / 5, \lambda / 3,2 \lambda / 3$, and $\lambda$. For each of these values, we have performed the Gaussian transition by enlarging the beam waist $w_{0}$ at a constant value of $L_{c}=35 \mu \mathrm{m}$, in order to illuminate from $n_{c}=\frac{\omega_{0}^{2}}{L_{c}^{2}}=0.25$ to $n_{c}=\frac{\omega_{0}^{2}}{L_{c}^{2}}=20$ correlation cells. The radial dependence of the complex field in the observation plane has been studied from $r=0$ to $2 w$.

\subsection{Speckle field distribution in the complex plane}

For a better qualitative understanding, we propose first to show the distribution of the on-axis speckle field in the complex plane in several characteristic regions of the circular Gaussian transition in Figure 2. Let us first focus our attention on the column corresponding to $n_{c}=20$ of Figure 2 that is expected to describe the near Gaussian regime. We see that with an increasing roughness value, the elliptic shape of the complex field distribution tends toward a circular one and that the mean value of the field vanishes. A non circular shape traduces the fact that the standard deviation of the real and imaginary part of the field are not equal. A non zero mean value of the field traduces a remaining specular component of the speckle field that is then called a partially developed speckle. Such variations of the shape of the distribution plot in the complex plane will be characterized with the degree of circularity $\zeta$ in Section 5.3.

Let us now look at the case $\left\{n_{c}=0.25 ; H_{R M S}=\lambda / 10\right\}$ that corresponds to a highly non circular and non Gaussian regime. We observe a very particular "moon shape" that evolves, with increasing roughness values at $n_{c}$ constant, toward a spiral shape that is clearly observable until we exceed a roughness value of $\lambda / 3$. From the same case $\left\{n_{c}=0.25 ; H_{R M S}=\lambda / 10\right\}$, at a constant roughness value but with an increasing number of illuminated correlation cells, the distribution evolves toward an elliptic shape. In fact, the spiral and elliptic shape distributions in near field exhibit some dependence between the real and imaginary part of the field, that will be studied in Section 5.4. In order to understand from a qualitative point of view the spiral shape of the complex field distribution, we have to notice that in such a strongly non Gaussian regime $\left(n_{c}=0.25\right)$, the laser 
beam illuminates much less than one correlation cell of the surface heights distribution. As a consequence, the dephasing mask encountered by the beam approaches the one of an inclined dephasing plane that tends to deviate this beam. For a given set of $L_{c o r r}$ and $H_{R M S}$ parameters, due the Gaussian distribution of the function $X(\eta, \xi)$, the slope of this inclined dephasing plane is going to vary among the different realizations of surfaces. Let consider first a realization giving rise to a null inclination of the dephasing plane. The non deviated beam will then remained centered on the on-axis ROI and this situation will give rise to a maximum detected amplitude with a low maximum value of dephasing (region $\mathrm{A}$ in Figure 2 for the case $\left\{n_{c}=0.25 ; H_{R M S}=\lambda / 5\right\}$ for example). Let now consider a realization corresponding to a maximum inclination of the dephasing plane. This situation will give rise to a maximum deviation of this beam that corresponds to the lowest detected amplitude on the on-axis ROI and to a strong maximum dephasing inside the beam (giving rise to a longer length of the spiral). This latter situation corresponds to region $\mathrm{B}$ of the same figure mentioned above.

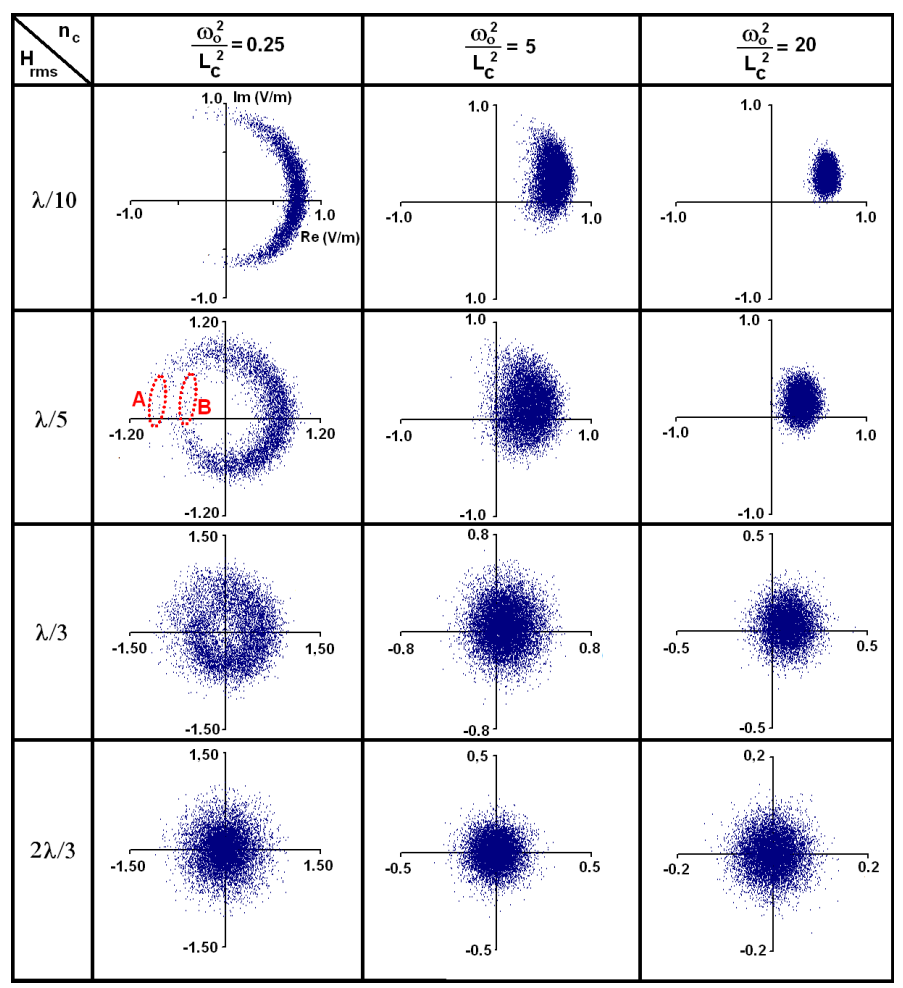

FIG. 2 Distribution plot of the on-axis speckle field in the complex plane presented at some characteristic regions of the circular Gaussian transition at the distance $z=$ $0.75 z_{R} . n_{c}$ represents the number of illuminated correlation cells and $H_{R M S}$ the roughness of the surface.

The column corresponding to $n_{c}=5$ is just presented to show a transition between the strongly non Gaussian case $\left(n_{c}=0.25\right)$ and the near Gaussian one $\left(n_{c}=20\right)$. Figure 3 represents the intensity distribution of the field around the optical axis for the situations corresponding to the ones of Figure 2. Especially, it exhibits the fact that the distribution plots showing a dependence between the real and imaginary part of the field in Figure 2 (cases of spiral and ellipse shapes distributions) are correlated with the presence of a strong remaining specular component of the speckle field. Moreover, we observe that for a high roughness value $(2 \lambda / 3)$ and in the near

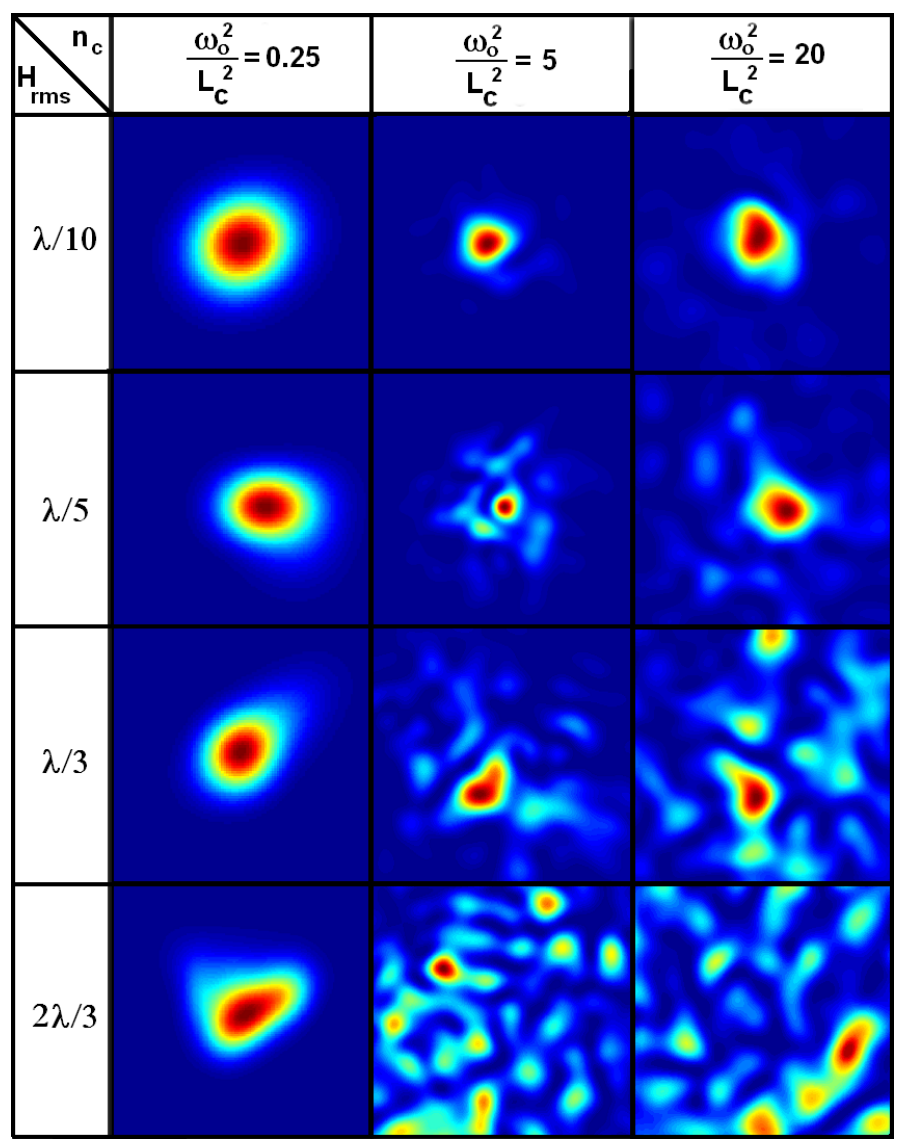

FIG. 3 Examples of intensity distributions in the observation plane at the distance $z=$ $0.75 z_{R}$, around the optical axis, at some characteristic regions of the circular Gaussian transition corresponding to the Figure 2. $n_{c}$ represents the number of illuminated correlation cells and $H_{R M S}$ the roughness of the surface.

Gaussian regime, the 20 correlation cells that contribute to the scattering are not sufficient to generate a stationary speckle field distribution. We would like to point out that all the plots of Figure 2 have undergone a suitable rotation in the complex plane in order to align the major and minor axis of ellipses (or similar structures) with the ones of the coordinate system (referenced to $(\mathrm{O}, \mathrm{X}, \mathrm{Y})$ in Figure 4). As so, instead of getting the values of $\sigma_{r}$ and $\sigma_{i}$ for the standard deviation of the real and imaginary part of the field, we get the values $\sigma_{x}$ and $\sigma_{y}$ in the newly rotated $(\mathrm{O}, \mathrm{X}, \mathrm{Y})$ coordinate system. We precise that such coordinate system is calculated for each ensemble of realizations of the scattered field corresponding to a given set of initial parameters $\left(H_{R M S}, L_{c}\right.$, illumination diameter and position of the observation ROI). This latter coordinate system will be used also for the calculation of the degree of circularity in Section 5.3. On the other hand, to characterize the degree of asymmetry of the field, another rotation will be performed in Section 5.4 to bring the mean value of the complex field on the real axis (referenced as $(\mathrm{O}, \mathrm{U}, \mathrm{V})$ on Figure 4), where the remaining tilt of the ellipses will characterize the correlation between the real and imaginary part of the speckle field. We would like to point out that such rotations don't modify any result concerning the intensity distribution of the speckle field, but allow to fix some reference coordinates systems suitable to follow the evolution of some properties about the real and imaginary part of the field in the circular Gaussian transition. A more detailed discussion is proposed in [6]. 


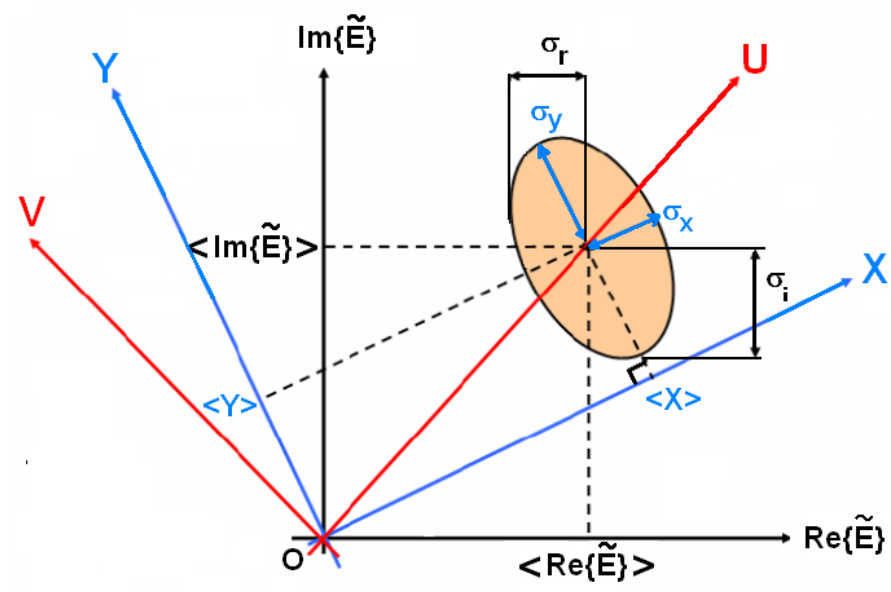

FIG. 4 Description of the coordinate systems used to characterize the statistical parameters of the speckle field. The coordinate system $(0, \operatorname{Re}\{\tilde{E}\}, \operatorname{Im}\{\tilde{E}\})$ where the calculated data are generated is rotated to the coordinate system $(0, X, Y)$ in order to calculate the standard deviation of the real and imaginary part of the field and is rotated to the coordinate system $(0, \mathrm{U}, \mathrm{V})$ for the calculation of the degree of asymmetry.

\section{2 speckle contrast}

We have plotted the radial dependence of the speckle contrast for different roughness values and illumination conditions in Figure 5 in the circular Gaussian transition. We observe that in agreement with previous results $[6,7,12]$, in the near Gaussian regime (generally defined approximately by $n_{c} \geq 5$ ), and for an increasing number of illuminated correlation cells, the contrast is always inferior to one or exhibits an asymptotic behaviour towards one. Note that for clarity, we have stopped the axis plot at $n_{c}=10$ and $n_{c}=5$ for the higher values of roughness $(\lambda / 3,2 \lambda / 3$ and $\lambda)$ because for each of these graphs, all the corresponding radial dependence curves were following roughly the same straight line until $n_{c}=20$. Moreover, we notice that the contrast gets always stronger when increasing the radial distance. This can be understood by the fact that when we move away from the optical axis, the remaining specular component vanishes and the only remaining part of the field is the diffuse one. In the case of a circular and near Gaussian speckle, as for example such a field generated by a surface characterized by $H_{R M S}=\lambda$ and $n_{c}>5$, the roughness is sufficiently strong to scatter the incident field without any remaining specular component. All the radial dependence curves are then superimposed : the scattered field tends to keep the same statistical properties in all directions.

Moreover, we see that some maxima around $n_{c}=0.25\left(L_{c}=\right.$ $2 \omega_{0}$ ) become observable for roughness values $\leq 2 \lambda / 3$. Such maxima in the speckle contrast has already been observed in the far field for objective speckle field $[7,12,19]$. However, we notice that for the special case of $\lambda / 10$ at the radial distance of $2 \omega$, the maximum is no more observable at least in the observed range of illumination conditions.

We would like to emphasize that we can observe a noticeable radial dependence of the contrast for roughness values between $\lambda / 3$ and $\lambda$ only in a strongly non Gaussian regime $\left(n_{c}<2\right)$. Such dependence exhibits moreover strong variations of contrast suitable for experimental discrimination. It is important to notice that without the use of the non Gaussian
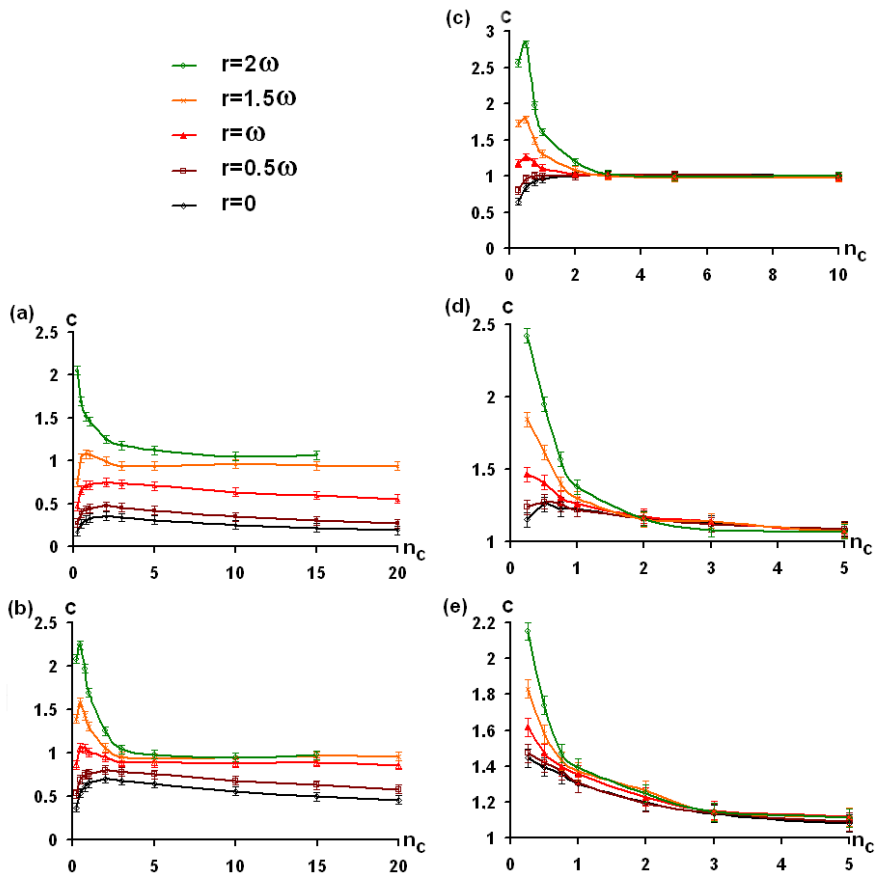

FIG. 5 Radial dependence of the speckle contrast in the circular Gaussian transition for roughness values of (a) $\lambda / 10$, (b) $\lambda / 5$, (c) $\lambda / 3$, (d) $2 \lambda / 3$ and (e) $\lambda$. Roughness values higher than $\lambda / 3$ can be discriminated by the radial dependence of the contrast only in a strongly non Gaussian regime when less than 2 correlation cells are illuminated.

regime, such range of roughness values exhibits a very similar contrast near one and prevents any experimental determination of the roughness value.

\subsection{Degree of circularity}

The degree of circularity of the complex speckle field can be defined by [6]:

$$
\zeta=\frac{\min \left(\sigma_{x}, \sigma_{y}\right)}{\max \left(\sigma_{x}, \sigma_{y}\right)}
$$

where $\sigma_{x}$ and $\sigma_{y}$ are the standard deviation of the real and imaginary part of the field in the rotated coordinate system $(\mathrm{O}, \mathrm{X}, \mathrm{Y})$ (Figure 4). This parameter is interesting to quantify the ellipticity of the field distribution in the complex plane presented in Section 5.1 and can have a non negligible influence on speckle contrast measurements. For example, Goodman has shown that the dip of contrast observed near the image plane of a lens first observed by J. Otsubo and T. Asakura[22] was due to a transition between circular and non circular statistics [23]. However, at this time, the behaviour of the degree of circularity in the circular Gaussian transition is known only in the Gaussian regime thanks to the work of Uozumi et al. [6]. Thus, we propose in Figure 6 the plot of the degree of circularity at four characteristic regions of the circular Gaussian transition, with its radial dependence, in order to show the main influence of non Gaussian statistics on the property of circularity. Moreover, in the near Gaussian region $\left(n_{c}=20\right)$ and for a roughness value of $\lambda / 2 \pi$, we could superimpose some previous data available from Uozumi et al. [6] that exhibit a good agreement with ours.

Let's notice that for clarity, we have stopped the plot to the roughness value of $2 \lambda / 3$ because the degree of circularity was 

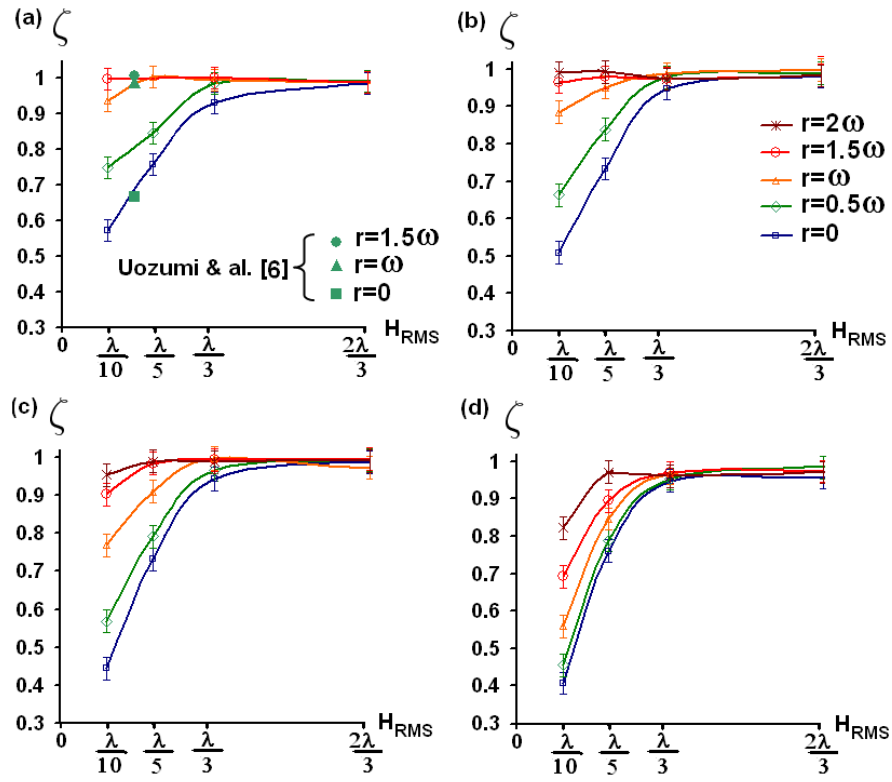

FIG. 6 Degree of circularity at four characteristic regions of the circular Gaussian transition where (a) $n_{c}=20$, (b) $n_{c}=3$, (c) $n_{c}=1$, and (d) $n_{c}=0.25$ correlation cells are illuminated. Results show good agreement with [6] in the Gaussian regime.

always 1 for higher values, within the uncertainty of our data. Decreasing the number of illuminated cells from 20 to 0.25 did not exhibit a significant variation in the degree of circularity until we reach about $n_{c}=3$. In the whole Gaussian transition, for all roughness values inferior or equal to $\lambda / 3$, we can observe a global decrease of the degree of circularity with a decreasing illuminated area and an increase of this degree with increasing radial distance. This latter observation is to correlate with the previously observed increase of contrast with the radial distance, due to the departure from the observation area containing the remaining specular component. Concerning a possible discrimination between different roughness values by using the Gaussian transition, we remark that an observation at the radial distance $r=\omega$ exhibits here the stronger difference of degree of circularity between the strongly non Gaussian regime $n_{c}=0.25$ and the near Gaussian one $n_{c}=20$ with a variation around 0.35 .

\subsection{Degree of asymmetry}

It is known from [6] that, in the Gaussian regime and for low roughness values, the speckle field exhibits a linear relation between its real and imaginary part, mainly present in the near field of the Gaussian illuminating beam and that neglecting this correlation results in a overestimation of the development of the speckle field. In order to study this dependence in the circular Gaussian transition, we define here, as in the previously cited reference, the degree of asymmetry $\mathcal{W}$ as the correlation coefficient between the real and imaginary part of the complex speckle amplitude in the coordinate system $(\mathrm{O}, \mathrm{U}, \mathrm{V})$ in which the mean imaginary part of the field is set to zero (see Figure 4). The corresponding analytical expression is then given by :

$$
\mathcal{W}=\frac{\langle\Delta U \Delta V\rangle}{\sigma_{U} \sigma_{V}}
$$

with $\Delta U=U-\langle U\rangle, \Delta V=V-\langle V\rangle, \sigma_{U}=\left\langle\Delta U^{2}\right\rangle$ and $\sigma_{V}=$ $\left\langle\Delta V^{2}\right\rangle$ where $\langle\cdots\rangle$ represents an average over the different realizations of the complex field.

With such a definition, in the $(\mathrm{O}, \mathrm{U}, \mathrm{V})$ coordinate system, the remaining tilt of the ellipse described by the equiprobability density function of the field will characterize the degree of asymmetry between the real and imaginary part of the speckle field. Especially, a case where the principal axes of the ellipse are parallel to the ones of the $(\mathrm{O}, \mathrm{U}, \mathrm{V})$ coordinate system corresponds to a null degree of asymmetry whereas on the opposite, a degree of asymmetry of one corresponds to a perfectly correlated set of real and imaginary values of the field and then to an ellipse that retracts to a straight line in the complex plane, making an angle of $\theta=\arctan \left(\sigma_{V} / \sigma_{U}\right)$ with the $\mathrm{U}$ axis. As for the degree of circularity, the data of [6] were available only in the Gaussian regime. Thus, in Figure 7, we have plotted such degree of asymmetry in 4 characteristic regions of the circular Gaussian transition and superimposed their results on the Gaussian region for their roughness value of $\lambda / 2 \pi$. Again, we can verify the good agreement between their data and ours in the Gaussian regime.

As a general behaviour, we notice in Figure 7 that the degree of asymmetry vanishes when the roughness value reaches about $\lambda / 3$ in all the Gaussian transition and that it tends to decrease with the radial dependence, the asymmetry of the field being contained within the remaining specular component.

Moreover, when describing the Gaussian transition from the near Gaussian regime $\left(n_{c}=15\right)$ to the highly non Gaussian one $\left(n_{c}=0.25\right)$, we observe a general decrease of the degree of asymmetry. However, some maxima, depending of the radial distance, could be observed during this transition. An example of such maximum can be observed within the four graphs of the Figure 7 for example for the radial distance $r=\omega$ where the degree of asymmetry increases from $n_{c}=15$ to $n_{c}=0.5$ and then decreases to $n_{c}=0.25$. Furthermore, we would like to point out that for roughness values superior to $\lambda / 3$, we can notice some remaining oscillations around zero which ampli-
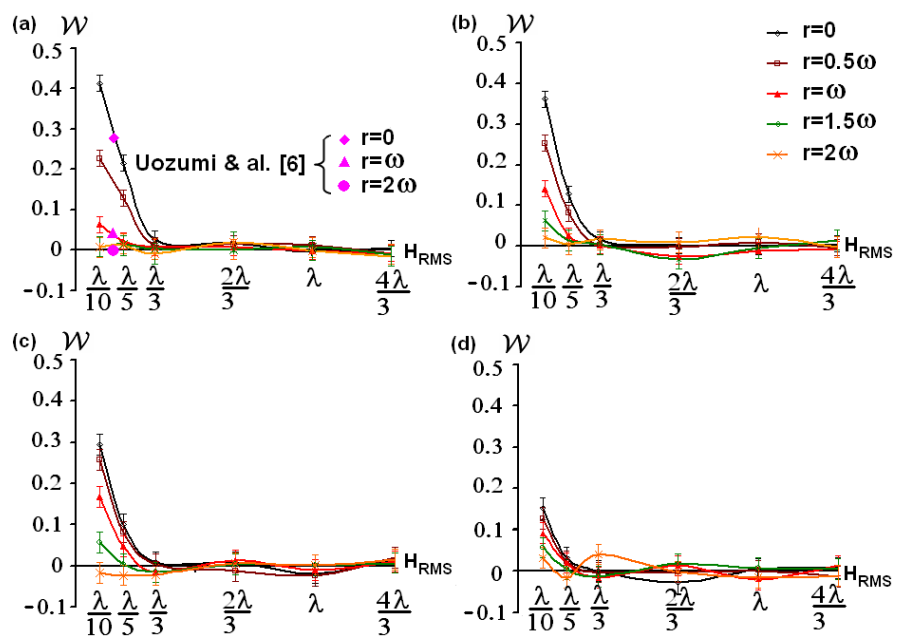

FIG. 7 Degree of asymmetry at four characteristic regions of the circular Gaussian transition where (a) $n_{c}=15$, (b) $n_{c}=1$, (c) $n_{c}=0.5$, (d) $n_{c}=0.25$ correlation cells are illuminated. Results show good agreement with [6] in the Gaussian regime. 
tude tends to increase with decreasing illuminated area. In order to explain this behaviour, we propose to come back to Figure 2 and focus our attention first on a low roughness value as $\lambda / 5$ for example. When we are in the near Gaussian regime where $n_{c}=20$ correlated cells are illuminated, we observe an elliptic shape of the distribution plot of the speckle field. This elliptic shape, once rotated in the $(\mathrm{O}, \mathrm{U}, \mathrm{V})$ coordinate system exhibits a strong remaining tilt traducing an important correlation between the real and imaginary part of the field. However, when we decrease the illuminated area, this linear dependence evolves toward a quadratic one, giving rise to the spiral shape in the complex plane (qualitatively explained in Section 5.1). The degree of asymmetry then reads this latter distribution as a succession of small linear dependencies giving alternatively positive and negative contributions to $\mathcal{W}$ depending on the sign of $\Delta U . \Delta V$ along the spiral. Then we can see in Figure $7(\mathrm{~d})$ that with increasing roughness value (that corresponds to an increasing length of the spiral), the degree of asymmetry exhibits some oscillations around zero which sign depends whether, for a given value of roughness, the sum of all the contributions of $\Delta U . \Delta V$ along the entire spiral length gives a global positive or negative sign.

As a conclusion of this section, the transition from Gaussian statistics to non Gaussian statistics is characterized for low roughness values, by a dependence between the real and imaginary part of the speckle field that evolves from a linear one to a quadratic one.

\section{CONCLUSION}

We have proposed a numerical approach to study optical speckle fields in the circular Gaussian transition where the contrast, the degree of circularity and the degree of asymmetry have been plotted with their radial dependence, in near field. Results of the numerical simulations have shown good agreement with previous works in the Gaussian regime when the central limit theorem can be applied and in the non Gaussian regime in the approximation of low roughness values. Moreover, for low standard deviation of surface heights distributions, we have shown that the linear dependence between the real and imaginary part of the speckle field in the Gaussian regime evolves toward a quadratic one in the non Gaussian regime, giving rise to spiral shapes distribution plots in the complex plane. Describing the circular Gaussian transition, we have demonstrated that the contrast and especially its radial dependence could be used to discriminate between different surface roughness values even in the range $[\lambda / 3, \lambda]$ by the use of a strongly non Gaussian regime when less than two correlation cells are illuminated. This result gives new insights for the experimental characterization of Gaussian surfaces : a decreasing laser spot radius can describe the Gaussian transition thus allowing the discrimination between roughness values in the full range $[0, \lambda]$. Let us remark that Gaussian statistics can be fully reached only in the case of an infinite number of scattering cells. A forthcoming article is underway concerning the characterization of the departure from Gaussian statistics inside the circular Gaussian transition studied in this paper. The numerical model proposed here can be extended to the study of other types of random surfaces, with different tilts of the illuminating beam, in near and far field.

\section{References}

[1] J. W. Goodman, Speckle phenomena in optics, theory and applications (Roberts and Company, 2006).

[2] J. W. Goodman, "Statistical properties of laser speckle patterns" in Laser speckle and related phenomena, J. C. Dainty, ed., 9-75 (Springer-Verlag, 1975).

[3] H. M. Pedersen, "On the contrast of polychromatic speckle patterns and its dependence on surface roughness" Opt. Acta 22, 15-24 (1975).

[4] E. Jakeman, and P. N. Pusey, "Non-Caussian fluctuations in electromagnetic radiation scattered by a random phase screen" J. Phys. A: Math. Gen. 8, 369-391 (1975).

[5] J. Ohtsubo, and T. Asakura, "Statistical properties of laser speckle produced in the diffraction field" Appl. Opt. 16, 17421753 (1977).

[6] J. Uozumi, and T. Asakura, "First-order intensity and phase statistics of Gaussian speckle produced in the diffraction region" Appl. Opt. 20, 1454-1466 (1981).

[7] H. M. Escamilla, "Speckle contrast in the diffraction field of a weak random-phase screen when the illuminated region contains a few correlation areas" Opt. Acta 30, 1655-1664 (1983).

[8] J. Uozumi, and T. Asakura, "The first-order statistics of partially developed non-Gaussian speckle patterns" J. Opt. 12, 177-186 (1981).

[9] H. Fujii, J. Uozumi, and T. Asakura, "Computer simulation study of image speckle patterns with relation to object surface profile" J. Opt. Soc. Am. 66, 1222-1236 (1976).

[10] J. Uozumi, H. Fujii, and T. Asakura, "Further computer simulation study of image speckle patterns with relation to object surface profile" J. Opt. Soc. Am. 67, 808-814 (1977).

[11] J. Uozumi, and T. Asakura, "Probability density functions of speckle intensity produced by weak diffusers" Opt. Acta 27, 1345-1360 (1980).

[12] H. Fujii, "Contrast variation of non-gaussian speckle" Opt. Acta 27, 409-418 (1980).

[13] R. S. Hansen, H. T. Yura, and S. G. Hanson, "First-order speckle statistics : an analytic analysis using ABCD matrices" J. Opt. Soc. Am. 14, 3093-3098 (1997).

[14] H. Kadano, T. Asakura, and N. Takai, "Roughness and correlation length determination of rough-surface objects using speckle contrast" Appl. Phys. B 44, 167-173 (1987)

[15] H. Kadano, T. Asakura, and N. Takai, "Roughness and correlationlength measurements of rough-surface objects using the speckle contrast in the diffraction field" Optik 80, 115-120 (1988).

[16] C. Cheng, C. Liu, N. Zhang, T. Jia, R. Li, and Z. Xu, "Absolute measurement of roughness and lateral-correlation length of random surfaces by use of the simplified model of image-speckle contrast" Appl. Opt. 41, 4148 (2002).

[17] A. K. Fung, and M. F. Chen, "Numerical simulation of scattering from simple and composite random surfaces" J. Opt. Soc. Am. A 2, 2274-2284 (1985).

[18] R. Grella, "Fresnel propagation and diffraction and paraxial wave equation" J. Opt. 13, 367-374 (1982). 
[19] P. J. Chandley, and H. M. Escamilla, "Speckle from a rough surface when the illuminated region contains few correlation areas: the effect of changing the surface height variance" opt. Commun. 29, 151-154 (1979).

[20] D. P. Qi, D. L. Liu, S. Y. Teng, N. Y. Zhang, and C. F. Cheng, "Morphological analysis by atomic force microscopy and light scattering study for random scattering screens" Acta. Phys. Sin.Ch. 49, 1260-1266 (2000).

[21] N. Y. Zhang, D. L. Liu, S. Y. Teng, D. P. Qi, and C. F. Cheng, “Ex- perimental study on the properties of autocorrelation function of image speckles produced by weak random screens" Acta. 0pt. Sin.-Ch. 21, 696-701 (2001).

[22] J. Ohtsubo, and T. Asakura, "Statistical properties of speckle intensity variations in the diffraction field under illumination of coherent light" Opt. Commun. 14, 30 (1975).

[23] J. W. Goodman, "Dependence of image speckle contrast on surface roughness" Opt. Commun. 14, 324-327 (1975). 